\title{
Multimorbilidade e Gravidade da Doença Medida pelo Índice de Charlson em Doentes Hospitalizados Durante o Ano de 2015: Estudo Transversal
}

\section{Multimorbidity and Disease Severity Measured by the Charlson Index in Portuguese Hospitalised Patients During the Year 2015: A Cross-Sectional Study}

\author{
Paula BROEIRO-GONÇALVES $\triangle^{1,2}$, Paulo NOGUEIRA ${ }^{3,4}$, Pedro AGUIAR ${ }^{5}$ \\ Acta Med Port 2019 Jan;32(1):38-46 - https://doi.org/10.20344/amp.9728
}

\section{RESUMO}

Introdução: A associação entre multimorbilidade e gravidade da doença não está bem estabelecida. Os objetivos foram caracterizar a multimorbilidade e determinar a gravidade da doença, bem como verificar se existe associação entre o número e natureza dos diagnósticos e o índice de Charlson.

Material e Métodos: Estudo transversal realizado através de dados exportados da base de dados de internamentos, durante o ano de 2015. O estudo incluiu 22 doenças crónicas: 15 previstas no índice de Charlson e sete condições médicas frequentes (hipertensão, obesidade, dislipidemia, osteoartrose, osteoporose, ansiedade e depressão). A análise foi realizada através do modelo linear generalizado, regressão logística binária. Na análise, utilizou-se a ferramenta IBM SPSS versão 24.0.

Resultados: Foram analisadas 800376 hospitalizações, das quais $42 \%$ correspondem a homens. A idade média da amostra foi de 59,8 anos, sendo maior nos homens (62,3 anos). O número médio de problemas por pessoa foi de 1,6, sendo superior nos homens $(1,8)$. A gravidade da doença também foi maior nos homens. O pior prognóstico esteve associado a seis ou mais condições por pessoa. O maior preditor de gravidade da doença foi o número de problemas, seguido da demência e diabetes.

Discussão: Os resultados parecem confirmar a diferença entre sexos quanto ao padrão de morbilidade. O número de condições por pessoa foi o maior preditor de gravidade da doença, particularmente a presença de seis ou mais condições por pessoa.

Conclusão: A principal limitação identificada foi o uso das mesmas condições médicas para medir a multimorbilidade e a gravidade da doença. Outros estudos e modelos de análise devem explorar a complexidade do fenómeno da multimorbilidade.

Palavras-chave: Comorbilidade; Índice de Gravidade de Doença; Multimorbilidade; Portugal

\section{ABSTRACT}

Introduction: The association between multimorbidity and disease severity is not well established. The objectives were to characterise multimorbidity and determine disease severity (trough Charlson), as well as to verify if there is an association between the number and type of disease and the Charlson index.

Material and Methods: A cross-sectional study based on exported data from the Portuguese National Health Service hospitalisations database, during the year 2015. The study included 22 chronic health conditions: 15 predicted in the Charlson index and seven frequent conditions (hypertension, obesity, dyslipidaemia, osteoarthritis, osteoporosis, anxiety and depression). The analysis was performed through the generalised linear model, considering binary logistic regression. In the analysis, the IBM SPSS version 24.0 tool was used. Results: The study analysed 800376 hospitalisations, from which $42 \%$ correspond to males. The average age of the sample was 59.8 years, being higher in men (62.3 years). The mean number of problems per person was 1.6, greater in men (1.8). Disease severity was also higher in males. The worst prognosis was associated with six or more conditions per person. The largest predictor of disease severity was the number of problems, followed by dementia and diabetes.

Discussion: The results seem to confirm the gender difference regarding morbidity pattern. The number of conditions per person was the greatest predictor of disease severity, particularly the presence of six or more conditions per person.

Conclusion: The major limitation was the use of the same medical conditions to measure multimorbidity and disease severity. Other studies and analysis models should explore the complexity of the multimorbidity phenomenon.

Keywords: Comorbidity; Multimorbidity; Portugal; Severity of Illness Index

\section{INTRODUÇÃO}

Os conceitos de multimorbilidade e comorbilidade referem-se à 'co-ocorrência de duas ou mais condições médicas numa pessoa', neste último, para além de uma doença índice. ${ }^{1}$ Os defensores do conceito de multimorbilidade tendem a concentrar-se na prática dos cuidados de saúde primários, onde a identificação de uma doença índice nem sempre é óbvia nem útil. ${ }^{1-4}$

As estimativas de prevalência de multimorbilidade variam devido à falta de consenso quanto à sua definição, modo como são colhidos os dados e ao número de diagnósticos considerados. ${ }^{4-10} \mathrm{~A}$ inexistência de padronização gera heterogeneidade o que torna os dados de prevalência

1. Unidade de Cuidados de Saúde Primários dos Olivais. Agrupamento de Centros de Saúde de Lisboa Central. Lisboa. Portugal.

2. Departamento de Medicina Geral e Familiar. Faculdade de Medicina. Universidade de Lisboa. Lisboa. Portugal.

3. Departamento de Saúde Ambiental. Faculdade de Medicina. Universidade de Lisboa. Lisboa. Portugal.

4. Laboratório de Biomatemática. Faculdade de Medicina. Universidade de Lisboa. Lisboa. Portugal.

5. Departamento de Saúde Pública. Escola Nacional de Saúde Pública. Universidade NOVA de Lisboa. Lisboa. Portugal.

$\triangle$ Autor correspondente: Paula Broeiro-Gonçalves. paulabroeiro@gmail.com

Recebido: 25 de setembro de 2017 - Aceite: 01 de outubro de 2018 | Copyright @ Ordem dos Médicos 2019 
não-comparáveis. ${ }^{4,7}$ Como referido, a frequência de multimorbilidade (MM) varia de acordo com a medida considerada: $43,7 \%$ para dois ou mais diagnósticos (MM2+), $27,4 \%$ (MM3+), 14,7\% (MM4+), 6,7\% (MM5+) e 2,8\% para seis ou mais diagnósticos coexistentes (MM6+). ${ }^{9}$

Uma outra questão crítica tem sido o facto do cálculo da estimativa de prevalência da multimorbilidade ser influenciado pelo número de diagnósticos considerados. ${ }^{4,6}$ Isto é, considerar quatro a sete diagnósticos conduziu a uma subestimação da prevalência, enquanto utilizar 12 ou mais diagnósticos gerou menor variabilidade. ${ }^{6}$ Por exemplo, um baixo número de diagnósticos (cinco diagnósticos) conduziu a um valor baixo de prevalência de multimorbilidade ( $0,3 \%$ na idade de 32,5 anos e $3,5 \%$ na idade de 75 anos) independentemente da dimensão amostral. ${ }^{6}$

Num estudo português, realizado em contexto de cuidados de saúde primários, na população adulta, nove em cada 10 participantes $(87,0 \%)$ tinham pelo menos um problema de saúde crónico, com uma média geral de 3,4 (3,6 em homens, 3,3 em mulheres). ${ }^{11} \mathrm{~A}$ multimorbilidade, medida como $\mathrm{MM} 2+$ esteve presente em $72,7 \%$ da amostra e se a medida era MM3+ foi de 57,2\%. ${ }^{11}$ Verificou-se uma associação significativa $(p<0,05)$ entre multimorbilidade e: género masculino, idade, residentes em áreas rurais, viver só, ter baixo nível social (escolaridade e rendimento) ${ }^{11}$ o que confirma os resultados de outros estudos que revelam associação entre multimorbilidade e gradientes sociais mais desfavorecidos. ${ }^{11-13}$

Atualmente tende a existir permutabilidade entre os conceitos de multimorbilidade e comorbilidade, no entanto, o índice de Charlson contínua a transmitir a noção de gravidade da comorbilidade associada a uma doença índice, definindo o peso total de doenças com impacto no indivíduo. ${ }^{1}$

Foram desenvolvidos índices com base no número e na informação sobre gravidade das condições médicas, resultando num valor agregado. Os índices de multimorbilidade têm sido utilizados para monitorização de serviços e comparar grupos de doentes ou prestadores de serviços. ${ }^{14}$ $\mathrm{O}$ índice de Charlson foi desenvolvido com base no risco relativo de morte e mede gravidade da doença utilizando morbilidades com diferente impacto no prognóstico (e.g., ponderação de seis para tumor sólido metastizado, dois para diabetes com complicações). ${ }^{15,16} \mathrm{O}$ índice global ou índice de Charlson permite aferir a comorbilidade (e.g., índice de três ou mais pontos significa grave comorbilidade) e predizer a mortalidade (e.g., índice de cinco ou mais pontos é preditor de morte a três anos, em $85 \%$ dos doentes). ${ }^{15}$ Tanto em cuidados primários como secundários, o índice de Charlson, um dos mais amplamente utilizados ${ }^{17}$ apresentou boa discriminação para sobrevivência a curto e longo prazo, prevendo a mortalidade com elevado poder discriminativo. ${ }^{18,19}$ Com os avanços da Medicina e o aumento da taxa de sobrevivência, problemas de saúde como a coronariopatia isquémica, a diabetes sem complicações ou a doença cerebrovascular não estiveram associadas a mortalidade levando mesmo a questionar a sua ponderação no índice de Charlson. ${ }^{16}$ Continua, no entanto, a considerar-se o índi- ce de Charlson um instrumento de prognóstico aplicado em saúde para medir a gravidade da doença. ${ }^{16,20}$

$\mathrm{O}$ índice de Charlson tem sido amplamente utilizado em estudos de resultados em saúde por permitir comparações válidas e o ajuste ao risco. ${ }^{21,22} \mathrm{O}$ ajuste ao risco é uma construção complexa que envolve fatores sociodemográficos do doente (e.g., idade), estabilidade clínica aguda ou gravidade da doença e foi criado como indicador da gravidade da doença. ${ }^{13,22}$ Tem crescido o interesse na utilização de bases de dados para estudar morbilidade, bem como o índice de Charlson aplicado a dados administrativos codificados segundo o CID-9..$^{21-23} \mathrm{Em}$ diversos estudos epidemiológicos o índice de Charlson foi utilizado para determinar a gravidade da doença num grupo populacional através de bases de dados. ${ }^{14,18-19,24}$ As bases de dados administrativas em investigação epidemiológica fornecem informação complementar aos estudos primários por corresponderem a dados da vida real, representando grandes amostras da população, sem viés de seleção. ${ }^{21}$

Conhecendo a validade do índice de Charlson enquanto instrumento de prognóstico que permite medir gravidade de doença, ${ }^{16,20}$ importa conhecer se existe associação entre multimorbilidade e gravidade de doença, considerando-se os diagnósticos e grupo etário.

Neste estudo, definiram-se como objetivos caracterizar a multimorbilidade em doentes internados nos hospitais do SNS e através do índice de Charlson determinar a gravidade da doença na transição da idade adulta até aos grandes idosos, bem como verificar se existe associação entre número e natureza de diagnósticos e índice de Charlson.

\section{MATERIAL E MÉTODOS}

Desenhou-se um estudo transversal, descritivo com componente analítica, através de dados exportados da base de dados Grupos de Diagnósticos Homogéneos (GDH).

Os GDH são um sistema de classificação de doentes internados em hospitais de agudos por grupos clinicamente coerentes e similares do ponto de vista do consumo de recursos. Permite definir operacionalmente os produtos de um hospital que cada doente recebe em função das suas necessidades e da patologia que o levou ao internamento. Permite pois identificar os diagnósticos por episódio de internamento e por indivíduo.

A população considerada no estudo foi a adulta (18 e mais anos) que teve pelo menos um internamento num hospital público durante o ano de 2015.

$\mathrm{O}$ índice de Charlson foi aplicado aos dados administrativos da base de dados $\mathrm{GDH}$, codificados segundo o CID-9 para determinar gravidade da doença à semelhança de outros estudos. ${ }^{16,21-23}$ A estratégia de cálculo do índice de Charlson a partir de dados provenientes de bases de dados $^{23}$ cumpriu as seguintes etapas: utilizaram-se os códigos CID10 atribuídos a cada diagnóstico de acordo com dados da literatura, ${ }^{25}$ os quais foram convertidos em códigos CID9 e posteriormente automaticamente ponderados de acordo com a pontuação atribuída a cada condição médica (Tabela 1). 
Tabela 1 - Correspondência entre diagnósticos e códigos CID9

\begin{tabular}{|c|c|c|}
\hline Condições Médicas & Ponderação & CID9 \\
\hline $\begin{array}{l}\text { Coronariopatia } \\
\text { isquémica }\end{array}$ & 1 & $\begin{array}{l}410410.00410 .01410 .02410 .10410 .11410 .12410 .2410 .20410 .21410 .22410 .3410 .30 \\
410.31410 .32410 .4410 .40410 .41410 .42410 .5410 .50410 .51410 .52410 .6410 .60 \\
410.61410 .62410 .7410 .70410 .72410 .8410 .80410 .82410 .9410 .90410 .91410 .92\end{array}$ \\
\hline $\begin{array}{l}\text { Insuficiência cardíaca } \\
\text { congestiva }\end{array}$ & 1 & $\begin{array}{l}428428.0428 .1428 .2428 .20428 .21428 .22428 .23428 .3428 .30428 .31428 .32428 .33 \\
428.4428 .40428 .42428 .43428 .9\end{array}$ \\
\hline $\begin{array}{l}\text { Doença vascular } \\
\text { periférica }\end{array}$ & 1 & $\begin{array}{c}440440.0440 .1440 .2440 .20440 .21440 .22440 .3440 .30440 .31440 .32440 .4440 .8 \\
440.9441441 .0441 .00441 .01441 .02441 .3441 .1441 .2441 .4441 .5441 .6441 .7441 .9 \\
442442.0442 .1442 .2442 .3442 .8442 .81442 .82442 .83442 .84442 .89442 .9443443 .0 \\
443.1443 .2443 .21443 .22443 .23443 .24443 .29443 .8443 .81443 .82443 .89443 .9\end{array}$ \\
\hline $\begin{array}{l}\text { Doença } \\
\text { Cerebrovascular }\end{array}$ & 1 & $\begin{array}{l}430431432432.0432 .1432 .9434434 .0434 .00434 .01434 .1434 .10434 .11434 .9 \\
434.90434 .91435435 .0435 .1435 .2435 .3435 .8435 .9436437437 .0437 .1437 .2437 .3 \\
437.4437 .5437 .6437 .7437 .8437 .9\end{array}$ \\
\hline Demência & 1 & $\begin{array}{l}290290.0290 .1290 .10290 .11290 .12290 .13290 .2290 .20290 .21290 .3290 .4290 .40 \\
290.41290 .42290 .43290 .8290 .9291 .2292 .82294294 .1294 .10294 .11294 .2294 .20 \\
294.21\end{array}$ \\
\hline DPOC & 1 & 490491491.0491 .1491 .2491 .20491 .21491 .22491 .8491 .9492492 .0492 .8 \\
\hline $\begin{array}{l}\text { Conectivite ou doença } \\
\text { do conjuntivo }\end{array}$ & 1 & $\begin{array}{l}517.2695 .4710710 .0710 .1710 .2710 .3710 .4710 .5710 .8710 .9714714 .0714 .1714 .2 \\
714.3714 .30714 .31714 .32714 .33714 .4714 .8714 .81714 .89714 .9725\end{array}$ \\
\hline Úlcera péptica & 1 & 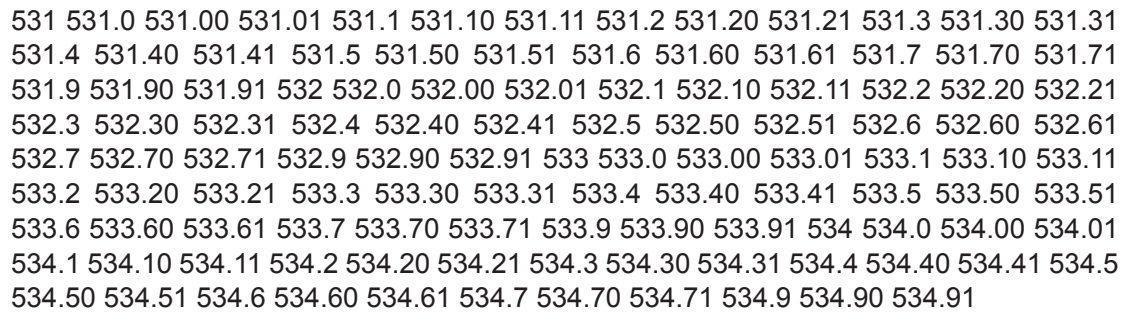 \\
\hline
\end{tabular}

Insuficiência hepática ligeira

Diabetes sem complicações

Diabetes com complicações

Hemiplegia ou Paraplegia

Insuficiência renal moderada a grave

Tumor sólido não metastizado ou Leucemia e Linfoma

Doença hepática moderada a grave

Tumor sólido metastizado

6

\subsection{1 .1571 .40571 .41571 .49571 .5571 .6571 .9}

\subsection{9 .00249 .01249 .10249 .2249 .20250 .0250 .00250 .01250 .02250 .03250 .20} 250.21250 .22250 .23

249.50249 .60249 .70250 .1250 .10250 .11250 .12250 .13250 .3250 .30250 .31250 .32 250.33250 .4250 .40250 .41250 .42250 .43250 .5250 .50250 .51250 .52250 .53250 .6

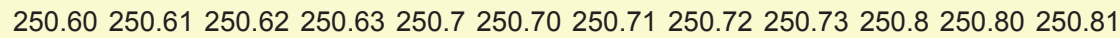
250.82250 .83250 .9250 .90250 .91250 .92250 .93357 .2362 .01362 .02362 .03362 .04 362.05362 .06362 .07366 .41 .

342342.0342 .00342 .01342 .02342 .1342 .10342 .11342 .12342 .8342 .80342 .81342 .82 $342.9342 .90342 .91342 .92344 .0344 .00344 .01344 .02344 .03 \quad 344.04344 .09344 .1$ 344.2344 .3344 .30344 .31344 .32344 .4344 .40344 .41344 .42344 .5438 .2438 .20438 .21 438.22438 .3438 .30438 .31438 .32438 .4438 .40438 .41438 .42

580.0580 .4580 .8580 .81580 .89580 .9582582 .0582 .1582 .2582 .4582 .81582 .89582 .9

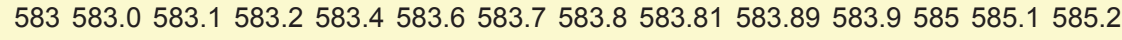
585.3585 .4585 .5585 .6585 .9586588588 .0588 .1588 .8588 .81588 .89588 .9

140140.0140 .1140 .3140 .4140 .5140 .6140 .8140 .9141141 .0141 .1141 .2141 .3141 .4 141.5141 .6141 .8141 .9142142 .0142 .1142 .2142 .8142 .9143143 .0143 .1143 .8143 .9 144144.0144 .1144 .8144 .9145145 .0145 .1145 .2145 .3145 .4145 .5145 .6145 .8145 .9 146146.0146 .1146 .2146 .3146 .4146 .5146 .6146 .7146 .8146 .9147147 .0147 .1147 .2 147.3147 .8147 .9148148 .0148 .1148 .2148 .3148 .8148 .9149149 .0149 .1149 .8149 .9

456.0456 .1456 .2456 .20456 .21571 .2571 .4571 .42571 .8572 .2572 .3572 .4572 .8573 .5

196196.0196 .1196 .2196 .3196 .5196 .6196 .8196 .9197197 .0197 .1197 .2197 .3197 .4 $197.5197 .6197 .7 \quad 197.8198198 .0198 .1 \quad 198.2 \quad 198.3 \quad 198.4 \quad 198.5198 .6198 .7198 .8$ 198.81198 .82198 .89199199 .0199 .1199 .2 
Para determinar a gravidade de doença através do índice de Charlson identificaram-se os 15 diagnósticos nele previstos, em que a diabetes e a insuficiência hepática têm dois níveis de gravidade, aos quais se adicionaram os problemas mais prevalentes (hipertensão arterial, obesidade, dislipidemia, osteoartrose, osteoporose, ansiedade e depressão) ${ }^{11,16}$ perfazendo um total de 22 diagnósticos (Tabela 1). Depois de definidos os diagnósticos a analisar identificaram-se os respectivos códigos na versão 9 da Classificação Internacional das Doenças (CID 9), classificação utilizada nos Grupos de Diagnósticos Homogéneos (GDH) (Tabela 1).

Da base de dados GDH correspondente a episódios de internamento, durante o ano de 2015, extraíram-se para cada episódio os dados sociodemográficos (idade e sexo) e os 22 diagnósticos de acordo com os códigos CID9 (Tabela 1).

Os dados foram exportados, para uma matriz Excel e posteriormente para a ferramenta estatística IBM SPSS versão 24.0 para o sistema operativo Mac OS a qual suportou a análise dos dados.

Dos dados exportados retiraram-se a população pediátrica ( 0 a $<18$ anos) e tendo em conta que um indivíduo pode ter um ou mais episódios de internamento num ano, à população adulta (idade igual ou superior a 18 anos) retiraram-se os duplicados tendo sido considerado o último episódio.

Os 22 diagnósticos foram dicotomizados correspondendo o valor 1 à presença e 0 à ausência. $O$ número de problemas por pessoa correspondeu ao somatório das condições médicas em presença. A multimorbilidade foi determinada de acordo com as definições de coexistência de dois ou mais diagnósticos (MM2+) até oito ou mais (MM8+).

Calculou-se o índice total Charlson considerando a ponderação atribuída aos diagnósticos na escala (Tabela 1). Este índice prevê o ajuste quanto à idade através de uma ponderação: 1 para o grupo 40 a $<50$ anos; 2 para o dos 50 a $<60$ anos; 3 para o dos 60 a $<70$ e 4 para o dos 70 e mais anos.

A análise descritiva teve em conta a idade, o número de problemas por pessoa e o índice de Charlson como variáveis numéricas. As variáveis numéricas foram categorizadas para facilitar a análise:

1. Grupos etários: jovens adultos [18 aos 39 anos], adultos [40 aos 54 anos], adultos na pré-reforma [55 aos 64 anos], $3^{\circ}$ idade [65 aos 74 anos], $4^{\text {a }}$ idade [75 aos 84 anos] e $5^{\mathrm{a}}$ idade [85 + anos].

2. Total de problemas por pessoa: de acordo com as definições de multimorbilidade, dois ou mais problemas por pessoa $(\mathrm{MM} 2+)$ até oito ou mais (MM8+).

3. Índice de Charlson por gravidade: calculado com ajustamento para a idade [cut-off 0 a $<5$; cut-off 5 a $<9$; cut-off $\geq 9$ ] ou sem ajustamento para a idade [cut-off < 5; cut-off $\geq 5$ ].

Tendo em conta a natureza binária das variáveis, a análise foi efetuada através do modelo linear generalizado (GLM) considerando-se a regressão logística binária.

A análise bivariável fez-se para cada um dos diagnósticos e para cada definição de multimorbilidade, como variáveis dependentes.

\section{Requisitos éticos}

A base de dados $\mathrm{GDH}$, com os dados de identificação pessoal encriptados, é disponibilizada aos alunos da ENSP mediante pedido de autorização superior e compromisso de confidencialidade.

O protocolo do estudo foi submetido à apreciação da Comissão de Ética para a Saúde da Administração Regional de Saúde de Lisboa e Vale do Tejo, da qual obteve parecer favorável.

\section{RESULTADOS}

Da base de dados GDH foram exportados 1026317 episódios dos quais 136574 corresponderam a episódios referentes à população pediátrica ( 0 aos < 18 anos). Corresponderam a episódios atribuíveis a adultos 800 376 após terem sido removidos $10 \%$ por duplicação, com o objectivo de a cada pessoa corresponder um episódio (o último) tal como referido na metodologia. Quanto ao sexo $42 \%$ (336 398) eram indivíduos do sexo masculino e

Tabela 2 - Ordenação por grupo etário e sexo

\begin{tabular}{|c|c|c|c|c|c|c|}
\hline Grupos etários & Masculino & & Feminino & & Total & Total \\
\hline $\begin{array}{l}\text { Adulto jovem } \\
\text { [18 aos } 39 \text { anos] }\end{array}$ & 43263 & $26,80 \%$ & 117971 & $73,20 \%$ & 161234 & $100,00 \%$ \\
\hline $\begin{array}{l}\text { Adultos } \\
\text { [40 aos } 54 \text { anos] }\end{array}$ & 58930 & $41,20 \%$ & 84224 & $58,80 \%$ & 143154 & $100,00 \%$ \\
\hline $\begin{array}{l}\text { Adultos na pré reforma } \\
\text { [55 aos } 64 \text { anos] }\end{array}$ & 61524 & $49,80 \%$ & 62018 & $50,20 \%$ & 123542 & $100,00 \%$ \\
\hline $\begin{array}{l}3^{\mathrm{a}} \text { idade } \\
{[65 \text { aos } 74 \text { anos] }}\end{array}$ & 74859 & $51,00 \%$ & 71783 & $49,00 \%$ & 146642 & $100,00 \%$ \\
\hline $\begin{array}{l}4^{\mathrm{a}} \text { idade } \\
{[75 \text { aos } 84 \text { anos] }}\end{array}$ & 70507 & $46,50 \%$ & 81210 & $53,50 \%$ & 151717 & $100,00 \%$ \\
\hline $\begin{array}{l}5^{\mathrm{a}} \text { idade } \\
{[85+\text { anos }]}\end{array}$ & 27315 & $36,90 \%$ & 46772 & $63,10 \%$ & 74087 & $100,00 \%$ \\
\hline Total & 336398 & $42,00 \%$ & 463978 & $58,00 \%$ & 800376 & $100,00 \%$ \\
\hline
\end{tabular}


$58 \%$ (463 978) do feminino. A média de idade da amostra foi de 59,8 anos, sendo superior nos homens (62,3 anos), e de 57,9 anos para as mulheres.

De forma a melhor compreender a distribuição, por grupo etário e sexo, dos indivíduos que tiveram pelo menos um internamento num hospital público, durante o ano de 2015, sintetizaram-se os dados na Tabela 2.

Conforme se pode observar existe um predomínio do sexo feminino em todos os grupos etários à exceção do grupo designado de terceira idade (entre os 65 e os 74 anos) grupo em que $51 \%$ eram homens.

O número médio de problemas por individuo foi de 1,6, superior no sexo masculino $(1,8)$ que no sexo feminino que foi de 1,44. A gravidade de doença através do índice de Charlson foi igualmente superior no sexo masculino $(1,11)$ enquanto no feminino foi de 0,74 e na amostra foi de 0,90 . A frequência da multimorbilidade para diferentes definições [entre dois ou mais problemas por pessoa (MM2+) a oito ou mais problemas por pessoa (MM8+)] revela um declínio de frequência em todos os grupos etários quando se progride de MM2+ para MM8+: 41,9\% (MM2+), 28,0\% (MM3+), 18,4\% (MM4+), 12,4\% (MM5+), 8,8\% (MM6+), $5,0 \%(\mathrm{MM} 7+)$ e $3,1 \%(\mathrm{MM} 8+)$.

Não se verificou uma associação estatisticamente significativa com a idade tanto para a magnitude (número de problemas), como para a gravidade (índice de Charlson) da multimorbilidade. As médias do número de problemas e a do índice de Charlson foram crescentes até ao grupo etário entre os 85 e os 89 anos. A partir dos 90 anos verificou-se um declínio para ambas as dimensões de multimorbilidade.

Atendendo a que a frequência das condições médicas decorre dos diagnósticos previstos na metodologia e dos problemas identificados na literatura como mais frequentes, analisou-se a sua associação ao sexo ajustado à idade.

Os diagnósticos associados ao sexo na análise bivariável ajustada à idade encontram-se na Tabela 3 por ordem decrescente do valor do odds ratio (OR). Destacam-se como problemas associados ao sexo masculino o tumor sólido não metastizado ou leucemia e linfoma, a doença hepática e doença vascular periférica. Ao sexo feminino estiveram associadas a osteoporose, conectivite ou doença do conjuntivo e a depressão.

A ansiedade (OR 2,016) e a depressão (OR 3,972), pelo OR mais elevado estiveram associadas $(p<0,001)$ a idades mais jovens (grupo etário - 45 a 54 anos), enquanto que a insuficiência cardíaca (OR 223,701), a insuficiência renal (OR 44,240) e a demência (OR 1864,620) às idades mais avançadas (grupo etário - 95 e mais anos), com o mesmo nível de significância $(p<0,001)$. A doença hepática (OR 9,929 ) e a doença oncológica metastizada (OR 12,629) revelaram associação aos grupos etários intermédios (grupo etário - 55 a 64 anos). Destaca-se a hipertensão arterial e a coronariopatia isquémica como diagnósticos com OR crescente a partir dos 65 anos. Os grupos etários dos mais jovens (até aos 45 anos) foram os que revelaram menor

Tabela 3 - Ordenação dos diagnósticos associados ao sexo

\begin{tabular}{|c|c|c|c|c|}
\hline $\begin{array}{l}\text { Diagnósticos } \\
\text { Sexo masculino }\end{array}$ & $p$ & OR & $\begin{array}{l}\text { Limite } \\
\text { inferior }\end{array}$ & $\begin{array}{l}\text { Limite } \\
\text { superior }\end{array}$ \\
\hline Tumor sólido não metastizado ou leucemia e linfoma & $<0,001$ & 5,276 & 4,838 & 5,753 \\
\hline Doença hepática & $<0,001$ & 2,813 & 2,719 & 2,910 \\
\hline Doença vascular periférica & $<0,001$ & 2,797 & 2,706 & 2,891 \\
\hline Coronariopatia isquémica & $<0,001$ & 2,205 & 2,132 & 2,280 \\
\hline DPOC & $<0,001$ & 2,175 & 2,124 & 2,227 \\
\hline Úlcera péptica & $<0,001$ & 1,973 & 1,876 & 2,075 \\
\hline Doença renal grave ou moderada & $<0,001$ & 1,483 & 1,457 & 1,510 \\
\hline Tumor sólido metastizado & $<0,001$ & 1,482 & 1,449 & 1,515 \\
\hline Diabetes & $<0,001$ & 1,311 & 1,295 & 1,327 \\
\hline Doença cérebro-vascular & $<0,001$ & 1,205 & 1,181 & 1,228 \\
\hline Dislipidemia & $<0,001$ & 1,150 & 1,137 & 1,163 \\
\hline Hipertensão arterial & $<0,001$ & 1,072 & 1,061 & 1,083 \\
\hline Insuficiência cardíaca congestiva & $<0,001$ & 1,065 & 1,048 & 1,083 \\
\hline \multicolumn{5}{|l|}{ Sexo feminino } \\
\hline Osteoporose & $<0,001$ & 6,738 & 6,304 & 7,203 \\
\hline Conectivite ou doença do conjuntivo & $<0,001$ & 2,775 & 2,632 & 2,925 \\
\hline Depressão & $<0,001$ & 2,766 & 2,706 & 2,827 \\
\hline Osteoartrose & $<0,001$ & 1,695 & 1,658 & 1,732 \\
\hline Ansiedade & $<0,001$ & 1,517 & 1,463 & 1,573 \\
\hline Obesidade & $<0,001$ & 1,384 & 1,360 & 1,408 \\
\hline Demência & $<0,001$ & 1,202 & 1,173 & 1,232 \\
\hline
\end{tabular}


número de diagnósticos associado a risco (OR > 1).

Realizou-se a análise multivariável dos diagnósticos associados a gravidade de doença (Charlson $\geq 5$ ) como variável dependente. Ajustou-se a totalidade dos diagnósticos ao sexo, à idade e ao número de problemas por pessoa. Conforme se pode observar na Tabela 4, o número de problemas por pessoa foi o maior preditor de gravidade de doença seguido da demência, diabetes, DPOC e Insuficiência cardíaca por ordem decrescente do valor de OR. O sexo feminino parece ter um efeito protetor $[p<0,001$; OR $0,807(0,773: 0,842)]$ e a idade efeito de risco $[p<0,001$; OR 1,713 (1,703: 1,724)].

Os diagnósticos incluídos pela sua frequência e não previstos no índice de Charlson não surgiram associados a gravidade de doença (Charlson $\geq 5$ ). Contudo, para a totalidade dos diagnósticos não incluídos na escala de Charlson (osteoartrose, osteoporose, ansiedade, depressão, hipertensão arterial, dislipedemia e obesidade) verificou-se associação significativa com o índice total $(p<0,001)$ (OR próximo do zero), com aparente efeito protetor.

Para aferir a associação entre as medidas de multimorbilidade e de gravidade de doença (índice de Charlson) realizou-se através do modelo GLM a análise bivariável. Quando se utilizaram as categorias de gravidade do índice de Charlson ajustado à idade: cut-off 5 a $<9$ (grave) e cut-off $\geq 9$ (muito grave) o comportamento da associação entre multimorbilidade e gravidade de doença foi diferente (Tabela 5) verificando-se a partir das seis condições médicas associação crescente com os dois cut-off de pior prognóstico, em particular com $\geq 9$.

Até à definição MM5+ (cinco ou mais diagnósticos por pessoa) não houve associação ao cut-off, do índice de Charlson, de maior gravidade $(\geq 9)$ e a tendência de associação com o cut-off 5 a $<9$ foi inconsistente. A partir de $\mathrm{MM} 6$ + o OR foi consistentemente progressivo para ambos os cut-off de gravidade.

\section{DISCUSSÃO}

Dos resultados deste estudo realçam-se o número médio de problemas por indivíduo superior no sexo masculino, ${ }^{1,8}$ bem como a gravidade de doença através do índice de Charlson. ${ }^{1,11}$ Confirma-se que frequência da multimorbilidade varia para diferentes definições [entre dois ou mais problemas por pessoa (MM2+) a oito ou mais problemas por pessoa $(\mathrm{MM} 8+)$ ] com declínio de frequência em todos os grupos etários quando se progride de $\mathrm{MM} 2+$ para $\mathrm{MM} 8+$ 41,9\% (MM2+), 28,0\% (MM3+), 18,4\% (MM4+), 12,4\% (MM5+), 8,8\% (MM6+), 5,0\% (MM7+) e 3,1\% (MM8+).

Tabela 4 - Ordenação dos diagnósticos associados ao índice de Charlson $\geq 5$ não ajustado para a idade na análise multivariável

\begin{tabular}{lcccc}
\hline Diagnósticos & $\boldsymbol{p}$ & OR & $\begin{array}{c}\text { Limite } \\
\text { inferior }\end{array}$ & $\begin{array}{c}\text { Limite } \\
\text { superior }\end{array}$ \\
\hline Total de problemas corrigido & $<0,001$ & 804,571 & 737,763 & 877,430 \\
Demência & $<0,001$ & 379,468 & 326,066 & 441,617 \\
Diabetes & $<0,001$ & 156,458 & 143,129 & 171,028 \\
DPOC & $<0,001$ & 111,830 & 100,183 & 124,832 \\
Insuficiência cardíaca congestiva & $<0,001$ & 103,997 & 94,112 & 114,921 \\
Tumor sólido não metastizado ou leucemia e linfoma & $<0,001$ & 89,627 & 70,070 & 114,643 \\
Conectivite ou doença do conjuntivo & $<0,001$ & 86,283 & 73,785 & 100,897 \\
Úlcera péptica & $<0,001$ & 70,029 & 57,713 & 84,974 \\
Doença cérebro-vascular & $<0,001$ & 64,745 & 58,290 & 71,915 \\
Doença vascular periférica & $<0,001$ & 40,663 & 35,344 & 46,783 \\
Doença renal grave ou moderada & $<0,001$ & 33,917 & 29,265 & 39,308 \\
Enfarte do miocárdio & $<0,001$ & 25,338 & 22,253 & 28,852 \\
Doença hepática & $<0,001$ & 24,303 & 20,324 & 29,062 \\
Hemiplegia ou paraplegia & $<0,001$ & 13,251 & 11,300 & 15,540 \\
\hline
\end{tabular}

Tabela 5 - Associação entre medidas de multimorbilidade e categorias de pior prognóstico do índice de Charlson ajustado à idade

\begin{tabular}{|c|c|c|c|c|c|c|c|c|}
\hline \multirow[b]{2}{*}{ Cut-off } & \multicolumn{4}{|c|}{$5 a<9$} & \multicolumn{4}{|c|}{$\geq 9$} \\
\hline & $p$ & OR & $\begin{array}{l}\text { Limite } \\
\text { inferior }\end{array}$ & $\begin{array}{l}\text { Limite } \\
\text { superior }\end{array}$ & $p$ & OR & $\begin{array}{l}\text { Limite } \\
\text { inferior }\end{array}$ & $\begin{array}{l}\text { Limite } \\
\text { superior }\end{array}$ \\
\hline MM2+ & $<0,001$ & 52,641 & 51,697 & 53,602 & n.s. & & & \\
\hline MM3+ & $<0,001$ & 29,495 & 29,099 & 29,896 & n.s. & & & \\
\hline MM4+ & $<0,001$ & 39,407 & 38,690 & 40,137 & n.s. & & & \\
\hline MM5+ & $<0,001$ & 76,959 & 74,325 & 79,687 & n.s. & & & \\
\hline MM6+ & $<0,001$ & 209,546 & 192,851 & 227,685 & $<0,001$ & 53836,701 & 48424,289 & 59841,703 \\
\hline MM7+ & $<0,001$ & 473,172 & 376,402 & 594,822 & $<0,001$ & 24346,826 & 19366,124 & 30608,496 \\
\hline MM8+ & $<0,001$ & 1097,052 & 579,187 & 2110,751 & $<0,001$ & 79684,008 & 41448,928 & 153189,513 \\
\hline
\end{tabular}


Estes resultados foram ligeiramente inferiores ao do estudo Australiano à exceção de $\mathrm{MM} 2+{ }^{9}$, provavelmente por serem relativos a dados de internamento. Nos mais idosos ( $\geq$ 90 anos) verificou-se um declínio para todas as medidas de multimorbilidade.

Os diagnósticos que estiveram associados ao sexo masculino (e.g., doença hepática ou DPOC) $)^{27,28}$ conferem o que se designou por coerência clínica decorrente do conhecimento empírico e confirmado por dados da literatura. ${ }^{29-36} \mathrm{O}$ mesmo se verificou pela associação ao sexo feminino dos diagnósticos: doença músculo-esquelética ${ }^{37}$ (osteoporose ${ }^{38}$ e conectivite) e doença mental ${ }^{37}$ (demência, ansiedade e depressão). Os problemas de saúde associados às idades mais avançadas tais como como a insuficiência cardíaca e a demência estão de acordo com os dados da literatura. ${ }^{39}$

A relação entre multimorbilidade e gravidade de doença não se encontra claramente estabelecida pelo que se utilizou o índice de Charlson (instrumento de prognóstico) como medida de gravidade da doença. ${ }^{16,20}$ Os índices, tal como o de Charlson, agregam a realidade complexa em indicadores únicos, como uma lista de doenças, com ponderações de acordo com o risco de morrer. Apesar da controvérsia gerada em torno da utilização de índices ${ }^{17}$ a aplicação do índice de Charlson confirmou a relevância clínica e reforça a sua pertinência na futura comparabilidade entre diferentes contextos. ${ }^{14,18,19} \mathrm{~A}$ associação de pior prognóstico ao sexo masculino, bem como dos diagnósticos de maior gravidade, parecem confirmar o 'paradoxo de género de morbilidade-mortalidade' sugerindo que nos homens os cuidados deveriam centrar-se em problemas de saúde fatais, como cancro, doenças cardíacas ou cerebrovasculares e nas mulheres, na gestão de condições de saúde relevantes para a limitação funcional (doença osteoarticular e mental). ${ }^{40,41}$

A validade dos resultados do nosso estudo poderá derivar da robustez conferida pela dimensão amostral, pela significância dos resultados, pelo rigor dos diagnósticos e pela sua coerência clínica anteriormente referida. Assume-se como rigor diagnóstico a inclusão de diagnósticos médicos profissionalmente precisos e a utilização de registos médicos classificadas de acordo com a Classificação Internacional de Doenças (CID). A utilização de registos médicos classificados de acordo com a CID é outro dos aspectos que contribuiu para a reprodutibilidade e validação externa dos resultados e comparabilidade da morbilidade entre os diferentes países ${ }^{17}$ ou contextos.

Questiona-se se a associação ao género masculino tem a ver com a fonte de dados associada a gravidade (dados de internamento), ao paradoxo de utilização de serviços relacionados com o sexo ou mesmo a características especificas da população Portuguesa. Isto porque os resultados revelam a associação da multimorbilidade ao sexo masculino como no estudo português realizado em CSP. ${ }^{11}$ Contudo, esses resultados não são consistentes com a meta-análise de Violan $\mathrm{C}$ et al, onde a maioria dos estudos incluídos revelaram associação com o sexo feminino. ${ }^{13}$
Em concordância com o número mínimo de condições médicas a incluir em estudos de multimorbilidade ${ }^{4}$ de forma a assegurar menor variabilidade ${ }^{6}$ o nosso estudo contemplou 22 condições de saúde crónicas, incluindo as mais frequentemente estudadas ${ }^{4}$ como a diabetes, a osteoartrose, a hipertensão ou o cancro. ${ }^{4} \mathrm{O}$ modelo de análise GLM e a utilização do índice de Charlson foram outras das estratégias metodológicas que tornam o nosso estudo reprodutível, contudo não extrapolável para a população por se tratar de dados de internamento.

O objectivo de aferir a associação entre as medidas de multimorbilidade e de gravidade de doença (índice de Charlson) através da análise bivariável no modelo GLM confirmou a associação entre qualquer medida de multimorbilidade (de $\mathrm{MM} 2+$ a MM8+) e o índice de Charlson. Quando se analisou a associação entre as medidas de multimorbilidade (de MM2+ a MM8+) e os três cut-off do índice de Charlson ajustado à idade $(<5,5$ a $<9$ e $\geq 9)$ o pior prognóstico esteve associado às definições de multimorbilidade superiores a seis e mais condições médicas. Apesar do aparente significado clínico o mesmo não foi encontrado na literatura. Questiona-se, no entanto, se não equivalerá ao conceito de multimorbilidade complexa, definida como a "co-ocorrência de três ou mais doenças crónicas afetando três ou mais sistemas diferentes numa pessoa". 9 Consideram-se necessários estudos futuros que clarifiquem o significado da definição de multimorbilidade de seis ou mais problemas (MM6+) como medida de gravidade. Há a referir que os resultados exponenciados no modelo GLM justificam a magnitude dos valores dos OR e respectivos intervalos de confiança (Tabela 5).

O número de problemas por pessoa foi o maior preditor de gravidade de doença seguido da demência, diabetes, DPOC e Insuficiência cardíaca por ordem decrescente do valor de OR. O que parece ser coerente com a diminuição da esperança média de vida associada à multimorbilidade em que cada condição crónica adicional diminui 1,8 anos de vida (variando de 0,4 menos anos com a primeira condição para 2,6 menos anos com a sexta condição). ${ }^{17}$

Como limitações do estudo identificam-se o desenho transversal e a natureza e a omissão de informação de natureza socioeconómica como escolaridade, rendimento ou rede social que nos dados da literatura se encontram associados a multimorbilidade de forma consistente. ${ }^{12,13} \mathrm{Ou}$ mesmo a omissão de fatores protetores como o elevado nível educacional, o estilo de vida saudável, a boa rede social e a prática de atividades de lazer, que atenuam o efeito da multimorbilidade. ${ }^{42}$

A limitação considerada major pelos autores foi ter-se utilizado as mesmas condições médicas em ambas as medidas de multimorbilidade e de gravidade (índice de Charlson). Todavia, este estudo deixa em perspectiva investigações futuras como estudos de desenho longitudinal que associem número e natureza das condições médicas e índice de Charlson ao longo do tempo em diferentes contextos (internamento, cuidados de saúde primários e população geral). Outros estudos e modelos de análise são 
necessários para que se possa explorar a complexidade do fenómeno multimorbilidade (social, mental e física) e clarificar a definição atual de multimorbilidade simplificada e aparentemente inadequada. ${ }^{17}$

Apesar das limitações acima identificadas, existem diversos aspetos que tornam único este estudo tais como:

- Os resultados serem provenientes de uma base de dados robusta, como a dos GDH, onde diagnósticos são codificados segundo a CID9 que permitiu a determinação da gravidade através do Índice de Charlson.

- A reprodutibilidade desta metodologia poderá vir a contribuir para a comparação entre serviços, unidades de saúde ou níveis de cuidados em investigações futuras.

- A dimensão amostral e a metodologia de análise permitirem confirmar a associação da gravidade da doença e da multimorbilidade ao sexo masculino.

- A associação entre a gravidade de doença e definição de multimorbilidade de seis ou mais condições médicas num individuo que poderá vir a ser uma medida simples de severidade.

\section{CONCLUSÃO}

A multimorbilidade e gravidade da doença estiveram associadas ao sexo masculino. Os resultados vêm confirmar a necessidade de intervenções em saúde adequadas ao sexo com os homens a sofrer de doenças mais graves

\section{REFERÊNCIAS}

1. Valderas JM, Starfield B, Sibbald B. Defining comorbidity: implications for understanding health and health services. Ann Fam Med. 2009;7:35763.

2. Broeiro P. Multimorbilidade e comorbilidade: duas perspectivas da mesma realidade. Rev Port Med Geral Fam. 2015;31:7-8.

3. Le Reste JY, Nabbe P, Manceau B, Lygidakis C, Doerr C, Lingner H, et al. The European General Practice Research Network presents a comprehensive definition of multimorbidity in family medicine and long term care, following a systematic review of relevant literature. J Am Med Dir Assoc. 2013;14:319-25.

4. Diederichs C, Berger K, Bartels DB. The measurement of multiple chronic diseases - a systematic review on existing multimorbidity indices. Journals Gerontol - Ser A Biol Sci Med Sci. 2011;66:301-11.

5. Stewart M, Fortin M, Britt HC, Harrison CM, Maddocks HL. Comparisons of multi-morbidity in family practice-issues and biases. Fam Pract. 2013;30:473-80.

6. Fortin M, Stewart M, Poitras M, Maddocks H. A systematic review of prevalence studies on multimorbidity: toward a more uniform methodology. Ann Fam Med. 2012;10:142-51.

7. Zellweger U, Bopp M, Holzer BM, Djalali S, Kaplan V. Prevalence of chronic medical conditions in Switzerland: exploring estimates validity by comparing complementary data sources. BMC Public Health. 2014; $14: 1157$.

8. Haregu T, Oldenburg B, Setswe G, Elliott J. Perspectives, constructs and methods In the measurement of multimorbidity and comorbidity : a critical review. Internet J Epidemiol. 2012;10:1-9.

9. Harrison C, Britt H, Miller G, Henderson J. Examining different measures of multimorbidity, using a large prospective cross-sectional study in Australian general practice. BMJ Open. 2014;4:e004694.

10. Mokraoui NM, Haggerty J, Almirall J, Fortin M, Schram M, Frijters D, et al. Prevalence of self-reported multimorbidity in the general population and in primary care practices: a cross-sectional study. BMC Res Notes. 2016;9:314.

11. Prazeres F, Santiago L. Prevalence of multimorbidity in the adult population attending primary care in Portugal: a cross-sectional study. BMJ Open. 2015;5:e009287.

12. Barnett K, Mercer SW, Norbury M, Watt G, Wyke S, Guthrie B. (doença oncológica, hepática e vascular) e as mulheres a condições médicas associadas a funcionalidade (doença osteoarticular e mental). Através do índice de Charlson, o número de problemas logo seguido da demência e da diabetes foram os maiores preditores de gravidade da doença. A definição de multimorbilidade, seis ou mais problemas por pessoa, de forma consistente esteve associada a gravidade de doença.

\section{PROTEÇÃO DE PESSOAS E ANIMAIS}

Os autores declaram que os procedimentos seguidos estavam de acordo com a Declaração de Helsínquia da Associação Médica Mundial.

\section{CONFIDENCIALIDADE DOS DADOS}

Os autores declaram ter seguido os protocolos do seu centro de trabalho acerca da publicação de dados.

\section{CONFLITO DE INTERESSES}

Os autores declaram que não há conflito de interesses na publicação deste artigo.

\section{FONTES DE FINANCIAMENTO}

Os autores declaram não ter recebido subsídios ou bolsas para a elaboração do artigo.

Epidemiology of multimorbidity and implications for health care research , and medical education : a cross-sectional study. Lancet. 2012;380:37-43.

13. Violan C, Foguet-Boreu Q, Flores-Mateo G, Salisbury C, Blom J, Freitag $\mathrm{M}$, et al. Prevalence, determinants and patterns of multimorbidity in primary care: A systematic review of observational studies. PLoS One. 2014;9:3-11.

14. Carey IM, Shah SM, Harris T, Dewilde S, Cook DG. A new simple primary care morbidity score predicted mortality and better explains between practice variations than the Charlson index. J Clin Epidemiol. 2013;66:436-44.

15. De Groot V, Beckerman H, Lankhorst GJ, Bouter LM. How to measure comorbidity: a critical review of available methods. J Clin Epidemiol. 2003;56:221-9.

16. Quan H, Li B, Couris CM, Fushimi K, Graham P, Hider P, et al. Updating and validating the charlson comorbidity index and score for risk adjustment in hospital discharge abstracts using data from 6 countries. Am J Epidemiol. 2011;173:676-82.

17. DuGoff EH, Canudas-Romo V, Buttorff C, Leff B, Anderson GF. Multiple chronic conditions and life expectancy. Med Care. 2014;52:688-94.

18. Crooks CJ, West J, Card TR. A comparison of the recording of comorbidity in primary and secondary care by using the Charlson Index to predict short-term and long-term survival in a routine linked data cohort. BMJ Open. 2015;5:e007974.

19. Brilleman SL, Salisbury C. Comparing measures of multimorbidity to predict outcomes in primary care: a cross sectional study. Fam Pract. 2013;30:172-8.

20. Charlson M, Szatrowski TP, Peterson J, Gold J. Validation of a combined comorbidity index. J Clin Epidemiol. 1994;47:1245-51.

21. Yurkovich M, Avina-Zubieta JA, Thomas J, Gorenchtein M, Lacaille D. A systematic review identifies valid comorbidity indices derived from administrative health data. J Clin Epidemiol. 2015;68:3-14.

22. Li B, Evans D, Faris P, Dean S, Quan H. Risk adjustment performance of Charlson and Elixhauser comorbidities in ICD-9 and ICD-10 administrative databases. BMC Health Serv Res. 2008;8:12.

23. Deyo RA, Cherkin DC, Ciol MA. Adapting a clinical comorbidity index for use with ICD-9-CM administrative databases. J Clin Epidemiol. 
1992;45:613-9.

24. Khan NF, Perera R, Harper S, Rose PW. Adaptation and validation of the Charlson Index for Read/OXMIS coded databases. BMC Fam Pract. 2010;11:1.

25. Ramiarina RA, Ramiarina BL, Almeida RM, Pereira WC de A Comorbidity adjustment index for the international classification of diseases, $10^{\text {th }}$ revision. Rev Saude Publica. 2008;42:590-7.

26. Gabinete de Estatísticas da União Europeia. Sustainable development in the European Union - 2015 monitoring report of the UE Sustainable Developmnt Strategy [Internet]. Luxembourg: Publications Office of the European Union; 2015. [consultado a 2017 fev 11]. Disponível em: http://ec.europa.eu/eurostat/documents/3217494/6975281/KS-GT-15001-EN-N.pdf.

27. Afonso AS, Verhamme KM, Sturkenboom MC, Brusselle GG. COPD in the general population: Prevalence, incidence and survival. Respir Med. 2011;105:1872-84.

28. Dal Negro RW, Bonadiman L, Turco P. Prevalence of different comorbidities in COPD patients by gender and GOLD stage. Multidiscip Respir Med. 2015;10:24.

29. Ferlay J, Steliarova-Foucher E, Lortet-Tieulent J, Rosso S, Coebergh $\mathrm{JW}$, Comber $\mathrm{H}$, et al. Cancer incidence and mortality patterns in Europe: estimates for 40 countries in 2012. Eur J Cancer. 2013;49:1374-403.

30. Malvezzi M, Carioli G, Bertuccio P, Rosso T, Boffetta P, Levi F, et al. European cancer mortality predictions for the year 2016 with focus on leukaemias. Ann Oncol. 2016;27:725-31.

31. Rehm J, Samokhvalov AV, Shield KD. Global burden of alcoholic liver diseases. J Hepatol. 2013;59:160-8.

32. Blachier $M$, Leleu $H$, Peck-Radosavljevic M, Valla DC, RoudotThoraval F. The burden of liver disease in Europe: a review of available epidemiological data. J Hepatol. 2013;58:593-608.

33. Townsend N, Wilson L, Bhatnagar P, Wickramasinghe K, Rayner M,
Nichols M. Cardiovascular disease in Europe: epidemiological update 2016. Eur Heart J. 2016;37:3232-45.

34. Bhatnagar P, Wickramasinghe K, Wilkins E, Townsend N. Trends in the epidemiology of cardiovascular disease in the UK. Heart. 2016;102:1945-52.

35. Sanchis-Gomar F, Perez-Quilis C, Leischik R, Lucia A. Epidemiology of coronary heart disease and acute coronary syndrome. Ann Transl Med. 2016;4:256

36. Ostan R, Monti D, Gueresi P, Bussolotto M, Franceschi C, Baggio G. Gender, aging and longevity in humans: an update of an intriguing/ neglected scenario paving the way to a gender-specific medicine. Clin Sci. 2016;130:1711-25.

37. McLean CP, Asnaani A, Litz BT, Hofmann SG. Gender differences in anxiety disorders: Prevalence, course of illness, comorbidity and burden of illness. J Psychiatr Res. 2011;45:1027-35.

38. Wright NC, Looker AC, Saag KG, Curtis JR, Delzell ES, Randall S, et al. The recent prevalence of osteoporosis and low bone mass in the United States based on bone mineral density at the femoral neck or lumbar spine. J Bone Min Res. 2014;29:2520-6.

39. Guzman-Castillo M, Ahmadi-Abhari S, Bandosz P, Capewell S, Steptoe $A$, Singh-manoux $A$, et al. Forecasted trends in disability and life expectancy in England and Wales up to 2025: a modelling study. Lancet Public Health. 2017;2:e307-13.

40. Chang WC, Lu FP, Lan TY, Wu SC. Multidimensional health-transition patterns among a middle-aged and older population. Geriatr Gerontol Int. 2012;13:571-9.

41. Luy M, Minagawa Y. Gender gaps - life expectancy and proportion of life in poor health. Health Reports. 2014;25:12-9.

42. Rizzuto D, Orsini N, Qiu C, Wang HX, Fratiglioni L. Lifestyle, social factors, and survival after age 75: population based study. BMJ. 2012;345:1-10. 